\title{
Efeitos genéticos e ambientais sobre o perímetro escrotal de animais da raça Caracu ${ }^{1}$
}

\author{
Maira Mattar ${ }^{2}$, João Ademir de Oliveira $^{3}$, Sarah Laguna Meirelles ${ }^{2}$, Sandra Aidar de Queiroz ${ }^{4}$ \\ ${ }^{1}$ Parte da Dissertação de Mestrado da primeira autora no programa de Genética e Melhoramento Animal - FCAV - Unesp/Jaboticabal, SP, \\ 2 Doutoranda - Programa de Pós Graduação - Zootecnia - FCAV - Unesp/Jaboticabal, SP. \\ ${ }^{3}$ Departamento de Ciências Exatas - FCAV - Unesp/Jaboticabal, SP. \\ ${ }^{4}$ Departamento de Zootecnia - FCAV - Unesp/Jaboticabal, SP. Bolsista do CNPq.
}

RESUMO - Neste trabalho foram avaliados os efeitos de idade (IDS) e peso aos 15 meses (P15) sobre medidas de perímetro escrotal ao sobreano de 1.892 machos da raça Caracu e estimada a herdabilidade desta característica. Utilizaram-se dois modelos de análise: em um dos modelos, foram incluídos o efeito de grupo de contemporâneos (GC) e, como covariável, a idade ao sobreano (efeitos linear e quadrático); e, no outro, o efeito de GC e, como covariável, o peso ao sobreano (efeitos linear e quadrático). A idade não teve efeito significativo no perímetro escrotal, o que está relacionado à homogeneidade dos animais nos grupos de contemporâneos, enquanto os efeitos linear e quadrático de P15 foram significativos sobre o perímetro escrotal, indicando grande influência do peso sobre a variação desta característica. A herdabilidade do perímetro escrotal foi estimada pelo método Bayesiano utilizando-se um modelo animal. O modelo incluiu os efeitos de GC e P15 (linear e quadrático) e os efeitos genéticos aditivo direto e residual. O valor médio estimado de herdabilidade do PE ao sobreano foi 0,38 e comprova que esta característica pode ser utilizada como critério de seleção para precocidade sexual em programas de melhoramento genético de animais da raça Caracu.

Palavras-chave: herdabilidade, idade ao sobreano, inferência bayesiana, peso ao sobreano, precocidade sexual

\section{Genetic and environment effects on scrotal circumference in Caracu cattle}

\begin{abstract}
Scrotal circumference (SC) is a trait of economic importance because it is related to reproduction and sexual precocity of males and females. This research aimed to evaluate the effects of yearling age (YA) and weight at 15 months of age (W15) on measures of SC using 1.892 records of Caracu young males. Two different models were compared. Both models included the contemporary group (CG) effect, but differed by using YA as a covariate (linear and quadratic components) in model 1 or by using W15 (linear and quadratic components) as the covariate in model 2. The YA effect was not significant on SC probably due to the homogeneity of the animals in contemporary groups. The linear and quadratic effects of W15 were significant on SC. Heritability was estimated by Bayesian method using the model including GC, W15 (linear and quadratic components) as fixed effects and animal and error as random effects. The mean estimate of heritability was 0.38 suggesting SC may be used as selection criteria for sexual precocity in breeding programs of Caracu cattle.
\end{abstract}

Key Words: Bayesian inference, heritability, sexual precocity, yearling age, weight at 15 months of age

\section{Introdução}

Os critérios de seleção baseados em precocidade sexual são, atualmente, pesquisados e incluídos em programas de melhoramento animal no intuito de otimizar a eficiência reprodutiva e, conseqüentemente, diminuir a idade de entrada dos animais em reprodução. No entanto, para melhorar a eficiência reprodutiva, além de analisar a precocidade por meio de indicativos nas fêmeas, as características medidas nos machos também podem contribuir para aumentar o progresso genético do rebanho. Desde a década de 60, diversos estudos com raças bovinas euro - péias têm abordado a utilização de características reprodutivas em machos, como o perímetro escrotal (PE), em programas de melhoramento. Apesar de a seleção para o aumento das medidas desta característica não trazer benefício econômico direto, esse aumento está geneticamente correlacionado, de modo favorável, a várias características reprodutivas, tanto de machos como de fêmeas, como idade ao primeiro parto (Martins Filho \& Lobo, 1991; Pereira et al., 2000) e peso corporal (Alencar et al., 1993; Bergmann et al., 1996; Pineda et al., 2000). Além disso, o perímetro escrotal é de fácil mensuração, de baixo custo e alta repetibilidade e, por isso, tem recebido grande atenção 
como possível critério de seleção de touros (Pratt et al.,1991), podendo melhorar mais rapidamente os rebanhos no aspecto reprodutivo.

Nos programas de melhoramento genético, tem-se recomendado como critério de seleção a utilização do perímetro escrotal ajustado tanto para idade (PEi) quanto para o peso corporal (PEp), uma vez que se procura não apenas outra medida do tamanho geral do animal, mas também uma medida da precocidade sexual dos machos (Dal Farra et al., 1998). Segundo Teixeira et al. (1998), é igualmente importante que os programas ofereçam aos produtores as medidas de PE corrigidas simultaneamente para peso e idade e somente para idade, pois pode haver diferentes prioridades quanto à precocidade sexual e à precocidade de crescimento, mesmo que essas características estejam correlacionadas. No entanto, a inclusão nas análises do efeito de idade juntamente com o de peso pode levar a não-significância de um deles, em virtude da inflação da variância decorrente da correlação entre esses dois efeitos (Dias et al., 2003).

As estimativas de herdabilidade do PE relatadas na literatura são altas, tanto na raça Nelore (Pereira et al., 2000; Ortiz Peña et al., 2001) como na Caracu (Queiroz et al., 2001), indicando a existência de variabilidade genética aditiva suficiente para obtenção de progresso genético na característica com a seleção de touros jovens.

A ênfase ao PE se deve ao fato de que, em parte, esta caraterística é favoravelmente correlacionada a características relacionadas à eficiência reprodutiva das fêmeas. No Brasil, Martins Filho \& Lôbo (1991) utilizaram 3.393 dados de touros jovens e 703 novilhas da raça Nelore e encontraram correlação genética entre perímetro escrotal e idade à primeira cria igual a $-0,44$, que indica que a seleção de touros com maior perímetro escrotal favoreceria a seleção de fêmeas com menor idade à puberdade, conferindolhes precocidade. Eler et al. (2004) encontraram correlação genética igual a 0,20 entre prenhez de novilha e perímetro escrotal, valor de pequena magnitude que não permite descartar a utilização do perímetro escrotal como critério de seleção para precocidade sexual.

No entanto, a obtenção de componentes de (co)variância para estimação de herdabilidade, pela abordagem clássica de modelos mistos, apresenta algumas limitações, como a dificuldade de se estabelecer intervalo de confiança adequado para esse parâmetro. Por isso, buscam-se novos procedimentos com propriedades de interesse e que forneçam estimativas mais acuradas. Uma opção para a solução de problemas relacionados às estimativas de parâmetros genéticos em populações animais é a utiliza- ção de métodos bayesianos, especialmente por meio do programa computacional MTGSAM (Van Tassel \& Van Vleck, 1995).

Uma diferença fundamental entre a abordagem clássica e a Bayesiana é que, pela clássica o parâmetro é considerado uma constante, embora desconhecida, enquanto, pela bayesiana, entende-se o parâmetro como uma variável aleatória que obedece a determinada distribuição de probabilidade, o que permite a construção de intervalos de credibilidade (o equivalente bayesiano de intervalo de confiança) utilizando-se métodos de simulação de Monte Carlo (MCMC), como o algoritmo amostrador de Gibbs.

Essa abordagem permite ainda a inclusão de informações pré-existentes (a priori) enriquecendo o processo de inferência. O conhecimento a priori dos parâmetros é modificado por meio da função de verossimilhança ou da distribuição amostral, que pode ser vista como a representação do que os dados denotam sobre os parâmetros. Combinando estas duas fontes de informações, a priori e verossimilhança, obtêm-se as chamadas distribuições a posteriori de todos os parâmetros.

Neste trabalho objetivou-se estudar os efeitos genéticos e ambientais que atuam sobre o perímetro escrotal de machos da raça Caracu. Avaliaram-se os efeitos da idade e do peso ao sobreano e estimou-se a herdabilidade dessa característica utilizando inferência Bayesiana.

\section{Material e Métodos}

Foram analisados dados de perímetro escrotal de machos de rebanhos da raça Caracu. O rebanho foi criado em 1893, quando foram adquiridos as primeiras matrizes e os primeiros touros. Os animais foram adquiridos até a década de 40, quando o criador passou a produzir seus próprios reprodutores. Até a década de 80, como a raça possui dupla aptidão, o rebanho foi direcionado para produção leiteira, de modo que a seleção dos animais foi baseada na produção das mães. Atualmente, a seleção dos machos é feita com um índice empírico com ponderação de $50 \%$ para as diferenças esperadas nas progênies (DEP) de produção de leite, $40 \%$ para peso e $10 \%$ para perímetro escrotal, ambos aos 15 meses de idade.

Os animais eram criados em regime pastoril e, nos meses mais frios, em virtude da deficiência na qualidade nutricional das pastagens, foram alimentados com silagem de milho (Zea mays, L). O manejo sanitário foi realizado por meio do controle e da prevenção de doenças, enquanto o manejo reprodutivo foi realizado com monta natural, com um touro para cada retiro e para cada lote de 50 fêmeas (em média). Os 
touros foram mantidos com as vacas no período de abril a fevereiro do ano seguinte, evitando-se nascimentos em dezembro e janeiro, quando a incidência de chuvas é maior.

As novilhas foram colocadas em reprodução com aproximadamente $350 \mathrm{~kg}$ e 18 meses de idade. Os touros, por sua vez, foram utilizados até os 5 anos de idade. Como os reprodutores foram produzidos na propriedade, evitou-se a realização de acasalamentos estreitos para que não ocorres seredução na produtividade atribuída à endogamia.

$\mathrm{O}$ arquivo de dados utilizado neste estudo continha informações de perímetro escrotal (PE), peso aos 15 meses (P15) e idade ao sobreano (IDS) de 1.892 machos, distribuídos em 120 grupos de contemporâneos (GC), além de dados de retiro de desmama, ano e estação de nascimento e rebanho de origem.

Os animais nasceram no período de 1999 a 2002. Os animais nascidos nos meses de setembro a novembro foram incluídos na estação 1 de nascimento; os nascidos em dezembro, janeiro e fevereiro foram incluídos na estação 2; os nascidos em março, abril e maio foram incluídos na estação 3; e os demais, nascidos em junho, julho e agosto, foram considerados da estação 4 de nacimento.

Inicialmente, os efeitos de idade (IDS) e peso ao sobreano (P15) sobre as medidas de perímetro escrotal (PE) foram analisados pelo método dos quadrados mínimos. Dois modelos foram utilizados, em ambos, foram incluídos o grupo de contemporâneos (GC) em classes, como efeito fixo, e, separadamente, a IDS como efeitos linear e quadrático (modelo 1) e P15 como efeitos linear e quadrático (modelo 2).

A estimação dos parâmetros genéticos foi realizada por inferência bayesiana, por modelo animal, utilizando-se o software MTGSAM (Van Tassel \& Van Vleck, 1995). O modelo incluiu os efeitos de grupo de contemporâneos e de peso ao sobreano (P15) (linear e quadrático) e os efeitos genético aditivo direto e residual para a obtenção das distribuições a posteriori das variâncias genética aditiva e residual do perímetro escrotal. A matriz de parentesco utilizada possuía 3.467 animais e o modelo empregado pode ser representado na sua forma matricial por: $\mathrm{Y}=\mathrm{X} \beta+\mathrm{Za}+\mathrm{e}$; em que: $\mathrm{Y}=$ vetor de observações $(\mathrm{PE}) ; \beta=$ vetor dos efeitos de GC e P15; a = vetor de efeitos genéticos aditivos; $\mathrm{X}$ e $\mathrm{Z}=$ matrizes de incidência associadas aos efeitos apropriados; e $=$ vetor de resíduos. Esse modelo considera as seguintes pressuposições: $\mathrm{E}(\mathrm{a})=0 ; \mathrm{E}(\mathrm{e})=0 ; \operatorname{Var}(\mathrm{a})=\mathrm{A} \sigma^{2}$; $\operatorname{Var}(\mathrm{e})=\mathrm{I}_{\mathrm{n}} \sigma_{\mathrm{e}}^{2}$; em que: $\mathrm{A}=$ numerador da matriz de parentesco $; \mathrm{I}=$ matriz identidade; $\mathrm{N}=$ número de registros; $\sigma_{\mathrm{a}}^{2}=$ variância genética aditiva direta; $\sigma_{\mathrm{e}}^{2}=$ variância residual. Um modelo alternativo incluindo apenas os efeitos de GC, genético aditivo e residualtambém foi utilizado para estimar a herdabilidade de PE.

Informações a priori foram tomadas como distribuições flat ou não-informativas (com a intenção de não caracterizar conhecimento prévio dos parâmetros) para os efeitos de GC e para a variância genética aditiva $\left(\sigma^{2}{ }_{\mathrm{a}}\right)$. Espera-se com a distribuição a priori que os dados sejam dominantes, ou seja, que a informação prévia tenha pouca influência nas estimativas.

Os valores iniciais de componentes de variância aditiva direta $\left(\sigma_{\mathrm{a}}^{2}\right)$ e residual $\left(\sigma_{\mathrm{e}}^{2}\right)$ de perímetro escrotal utilizados no programa MTGSAM foram iguais a 1,56 e 2,50 $\mathrm{cm}^{2}$, respectivamente. Iniciou-se então a geração de uma seqüência de vetores compostos pelos componentes das variâncias aditiva direta e residual $\left(\sigma_{\mathrm{a}}^{2} ; \sigma_{\mathrm{e}}^{2}\right)$ obtidos da distribuição a posteriori conjunta dos mesmos.

O comprimento das cadeias de Markov utilizado na análise foi de 307.000 ciclos (vetores), com descarte dos 7.000 primeiros vetores (burn-in), considerando vetores a cada 300 ciclos, o que proporcionou 1.000 vetores simulados para a análise. Esses valores foram obtidos por meio de uma análise prévia dos vetores $\left(\sigma_{\mathrm{a}}^{2}, \sigma_{\mathrm{e}}^{2}\right)$, por meio do programa Gibanal, sob sistema operacional DOS, desenvolvido por Van Kaam (1997).

Os valores das herdabilidades do PE foram obtidos a partir da amostra de variâncias genéticas aditivas $\left(\sigma^{2}{ }_{a}\right)$ e de variâncias residuais $\left(\sigma_{\mathrm{e}}^{2}\right)$ vetor a vetor. A herdabilidade para cada vetor foi obtida por: $h^{2}=\sigma^{2}{ }_{a} /\left(\sigma^{2}{ }_{a}+\sigma^{2}{ }_{e}\right)$. Após a obtenção dos valores das herdabilidades para todos os vetores da amostra, calculou-se a média de todos os valores gerados, ou seja, a estimativa Bayesiana desse parâmetro.

O intervalo de credibilidade (IC) utilizado na análise Bayesiana foi formado pela eliminação dos $5 \%$ menores e dos 5\% maiores valores das distribuições a posteriori dos parâmetros.

A convergência foi verificada graficamente (Gelfand \& Smith, 1990) pelo teste Z de Geweke (Gamerman, 1997).

\section{Resultados e Discussão}

$\mathrm{Na}$ análise de variância de PE, em que foi considerado o efeito de idade do animal (IDS), o valor do coeficiente de variação foi baixo $(7,67 \%)$, evidenciando homogeneidade nos dados (Tabela 1). O coeficiente de determinação foi igual a 43,04\% e comprova que o modelo utilizado explicou apenas parte da variação da característica. Tanto o efeito linear quanto o quadrático de IDS não influenciaram significativamente o perímetro escrotal. Possivelmente, a nãosignificância deste efeito neste estudo pode ser atribuída 
Tabela 1 - Resumo da análise de variância do perímetro escrotal de bovinos da raça Caracu, de acordo com peso aos 15 meses (P15) e idade ao sobreano (IDS)

Table 1 - Summary of analysis of variance for scrotal circumference of Caracu cattle according to different models including yearling weight (P15) or yearling age (IDS) fixed effects

\begin{tabular}{|c|c|c|c|c|c|}
\hline \multirow[b]{2}{*}{${ }^{1} \mathrm{FV}$} & \multicolumn{2}{|c|}{$\begin{array}{c}\text { Modelo } 1 \\
\text { Model } 1\end{array}$} & \multirow[b]{2}{*}{${ }^{1} \mathrm{FV}$} & \multicolumn{2}{|c|}{$\begin{array}{c}\text { Modelo } 2 \\
\text { Model } 2\end{array}$} \\
\hline & ${ }^{2} \mathrm{GL}$ & ${ }^{3} \mathrm{QM}$ & & ${ }^{2} \mathrm{GL}$ & ${ }^{3} \mathrm{QM}$ \\
\hline${ }^{4} \mathrm{GC}$ & 119 & $27,627 * *$ & ${ }^{4} \mathrm{GC}$ & 119 & $42,4257 * *$ \\
\hline $\mathrm{P} 15(\mathrm{YW}){ }^{5} \mathrm{~L}$ & 1 & $51,641 * *$ & $\operatorname{IDS}(\mathrm{YA}){ }^{5} \mathrm{~L}$ & 1 & 4,8612 \\
\hline $\mathrm{P} 15(\mathrm{YW}){ }^{6} \mathrm{Q}$ & 1 & $140,3767 * *$ & IDS (YA) ${ }^{6} \mathrm{Q}$ & 1 & 1,6203 \\
\hline Resíduo & 1.770 & 4,03467 & Resíduo & 1.770 & 5,62010 \\
\hline${ }^{7} \mathrm{R}^{2}(\%)=59,11$ & & & ${ }^{7} \mathrm{R}^{2}(\%)=43,04$ & & \\
\hline${ }^{8} \mathrm{CV}(\%)=6,50$ & & & ${ }^{8} \mathrm{CV}(\%)=7,67$ & & \\
\hline
\end{tabular}

${ }^{1}$ Fonte de variação(source of variation), ${ }^{2}$ grau de liberdade (degree offreedom), ${ }^{3}$ quadrado médio (mean square), ${ }^{4}$ grupo de contemporâneos (contemporarygroup), ${ }^{5}$ linear (linear), ${ }^{6}$ quadrático (quadratic), ${ }^{7}$ coeficiente de determinação (coefficient of determination), ${ }^{8}$ coeficiente de variação (coefficient of variation), ${ }^{\star \star} \mathrm{P}<0,001$.

à formação dos GC, composto, entre outros efeitos, pelo ano e pela estação de nascimento do animal. Esses componentes do GC na análise, indiretamente, corrigiram o efeito da idade do animal sobre o perímetro escrotal, o que reflete a não-significância do efeito da idade do animal. Resultados similares foram relatados por Ortiz Peña et al. (2001) e Dias et al. (2003), que também não verificaram significância estatística $(\mathrm{P}>0,05)$, respectivamente, do efeito quadrático e do efeito linear e quadrático da idade do animal sobre perímetro escrotal.

No resultado da análise de variância do PE considerando P15 como covariável, o coeficiente de variação também foi de baixa magnitude $(6,50 \%)$. No entanto, o coeficiente de determinação foi maior (59\%), evidenciando que o modelo explicou em maior proporção a variação da característica estudada. Os efeitos linear e quadrático de P15 sobre PE foram estatisticamente significativos $(\mathrm{P}<0,001)$, indicando que o peso ao sobreano teve grande influência nas medidas de PE dos animais.

Em ambas as análises, o GC influenciou significativamente $(\mathrm{P}<0,001)$ o perímetro escrotal dos animais. A equação de regressão do PE sobre o peso ao sobreano, ajustada para o efeito de GC, foi: $\hat{Y}=8,751+0,111 x-0,00011 x^{2}$; em que: $\hat{\mathrm{Y}}=$ perímetro escrotal ao sobreano, em $\mathrm{cm} ; \mathrm{x}=$ peso ao sobreano, em kg.

Pela equação de regressão (Figura 1), verificou-se variação máxima de aproximadamente $20 \mathrm{~cm}$ nas medidas do PE predito entre animais com menor e maior peso ao sobreano, evidenciando que peso ao sobreano constitui importante fonte de variação de PE.

O perímetro escrotal é genética e positivamente correlacionado ao peso corporal em várias idades. Pesquisadores (Meyer et al., 1991; Alencar et al., 1993; Bergmann et al., 1996) sugeriram que a seleção para aumento do PE deve ocasionar, por resposta correlacionada, aumento de

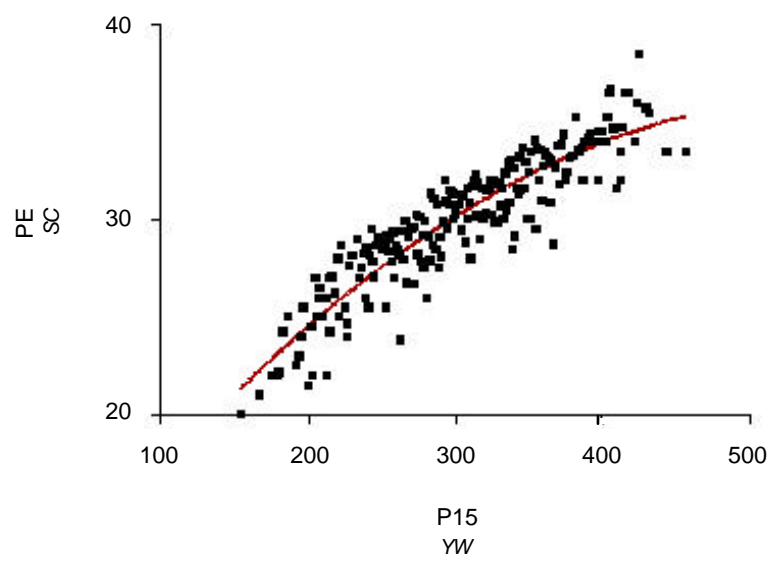

Figura 1 - Crescimento do perímetro escrotal (PE), em $\mathrm{cm}$, de bovinos Caracu em função do peso ao sobreano (P15), em kg.

Figure 1 - Regression of scrotal circumference growth $(\mathrm{SC}, \mathrm{cm})$ on yearling weight $(\mathrm{YW}, \mathrm{kg})$ in Caracu cattle.

peso corporal em várias idades, o que nem sempre é desejável. Nesses casos, segundo Ortiz Peña et al. (2001), é possível diminuir esta resposta correlacionada procedendo-se à seleção com base no PE ajustado para idade e peso corporal (PEip). Queiroz et al. (2001) encontraram correlação do PEip e peso aos 15 meses de idade igual a $0,11 \mathrm{em}$ bovinos Caracu. Esse pequeno valor de correlação genética evidenciou que a remoção dos efeitos de idade e peso do animal permitiu que o PE pudesse ser usado de modo mais adequado como critério reprodutivo. No entanto, neste estudo, o efeito da idade ao sobreano sobre o perímetro escrotal não foi estatisticamente significativo, o que tornou desnecessária a correção do PE para esta fonte de variação.

A média a posteriori da herdabilidade de PE foi igual a 0,38 (Tabela 2). Valores da magnitude do estimado neste estudo indicam que a seleção para PE seria modera- 
Tabela 2 - Médias a posteriori dos componentes de variância e herdabilidade do perímetro escrotal de machos da raça Caracu

Table 2 - Mean a posteriori of variance components and heritability for scrotal circumference of Caracu cattle

\begin{tabular}{lccccc}
\hline $\begin{array}{l}\text { Estimativa } \\
\text { Estimate }\end{array}$ & ${ }^{4} \mathrm{~N}$ & $\begin{array}{c}\text { Média } \\
\text { Mean }\end{array}$ & ${ }^{5} \mathrm{IC}$ & $\begin{array}{c}\text { Mínimo } \\
\text { Minimum }\end{array}$ & $\begin{array}{c}\text { Máximo } \\
\text { Maximum }\end{array}$ \\
\hline${ }^{1} \sigma^{2}$ & 1.000 & 1,59 & {$[1,07 ; 2,15]$} & 0,62 & 3,08 \\
${ }^{2} \sigma^{2}{ }^{\mathrm{a}}$ & 1.000 & 2,49 & {$[2,06 ; 2,89]$} & 1,43 & 3,41 \\
${ }^{3} \mathrm{~h}^{2}$ & 1.000 & 0,38 & {$[0,27 ; 0,50]$} & 0,15 & 0,66 \\
\hline
\end{tabular}

${ }^{1}$ Variância genética aditiva (additive genetic variance), ${ }^{2}$ variância residual (residual variance), ${ }^{3}$ herdabilidade (heritability), ${ }^{4}$ tamanho da amostra (sample size), ${ }^{5}$ intervalo de $90 \%$ de credibilidade $(90 \%$ credibility interval).

damente eficiente para promover ganho genético na precocidade sexual dos animais da raça Caracu.

Bordon \& Brinks (1986) comentaram que o ajuste do perímetro escrotal pelo peso corporal (PC) removeria parte do componente genético aditivo, causando diminuição do PC e da herdabilidade. Entretanto, a estimativa média do PE desconsiderando os efeitos de peso e idade aos 15 meses obtida neste estudo foi igual a 0,39 , muito similar à obtida corrigindo-se a característica para peso corporal $(0,38)$.

Em análises bayesianas são utilizados os intervalos de credibilidade (IC) (Tabela 2), que, para a herdabilidade, variaram de 0,27 a 0,50 , indicando que o valor real do parâmetro se encontra neste intervalo e pode ser considerado de magnitude moderada a alta.

$\mathrm{O}$ algoritmo de Gibbs produz uma cadeia de amostra de tantas iterações quanto especificadas. A monitoração da convergência das cadeias de Markov pode ser feita por meio da análise gráfica dos valores gerados da distribuição a posteriori. Se a trajetória de uma única cadeia ao longo das iterações é próxima de um valor constante, conclui-se pela convergência da cadeia (Gamerman, 1996). A convergência foi confirmada pelo escore de $\mathrm{Z}$ do teste de Geweke (Gamerman, 1997), igual a 0,11. Segundo Blasco et al. (2003), escores de $\mathrm{Z}$ do teste de Geweke indicam que a convergência foi alcançada dentro do intervalo $-1,96<Z<1,96$.

As variâncias genéticas aditivas obtidas na amostra não apresentaram grande dispersão (Figura 2), ou seja, as oscilações mantiveram-se estáveis, evidenciando que o valor considerado para o burn-in na análise foi confiável e permitiu a convergência da cadeia (Gelfand \& Smith, 1990).

Estimativas de herdabilidade do $\mathrm{PE}$ superiores à obtida neste trabalho foram relatadas por Ortiz Pena et al. (2001) e Dias et al. (2003), em estudo com dados de animais da raça Nelore (0,47 e 0,42, respectivamente), enquanto Queiroz et al., (2001), em pesquisa com um dos rebanhos de bovinos

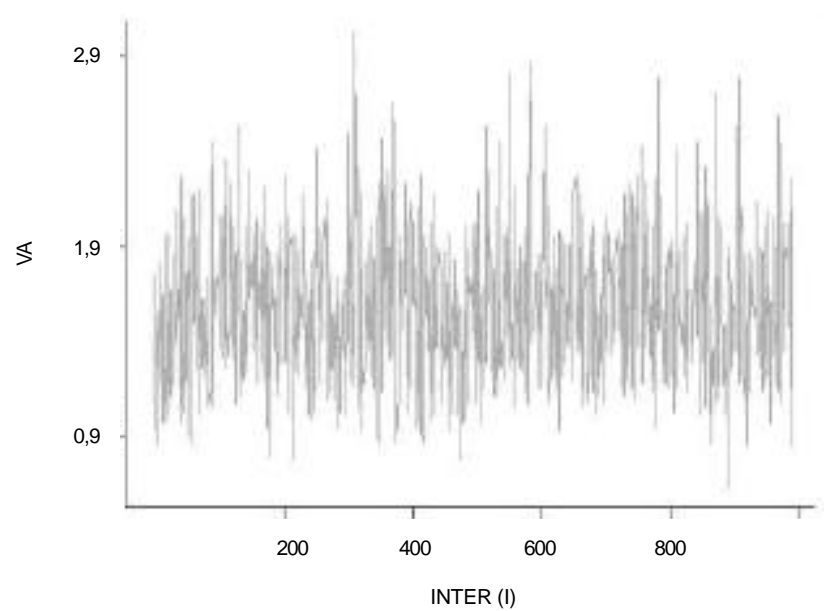

Figura 2 - Estimativas a posteriorida variância genética aditiva (VA) do perímetro escrotal de bovinos Caracu por ciclo (ITER I).

Figure 2 - A posteriori estimates of additive genetic variance for scrotal circumference of Caracu cattle.

Caracu deste estudo, obtiveram estimativa de 0,41 pelo método da máxima verossimilhança. Estimativas de herdabilidade de magnitude ainda maior foram obtidas por Bourdon \& Brinks (1986) para PE corrigido para o peso aos 12 e aos 18 meses de idade (0,60 e 0,71, respectivamente).

\section{Conclusões}

O peso do animal consistiu em importante fonte de variação do perímetro escrotal (PE) ao sobreano e deve ser considerado nos modelos. A magnitude de sua estimativa de herdabilidade sugere a existência de variabilidade genética aditiva suficiente para PE se utilizado como critério de seleção.

\section{Agradecimento}

Ao Zootecnista M.Sc. André Stein Carvalho Dias, pela cessão dos dados utilizados nesta pesquisa. Ao Professor Adhemar Sanches, pelo auxílio na compreensão da metodologia Bayesiana.

\section{Literatura Citada}

ALENCAR, M.M.; BARBOSA, P.F.; BARBOSA, R.T. et al. Parâmetros genéticos para peso e perímetro escrotal em touros da raça Canchim. Revista Brasileira de Zootecnia, v.22, n.4, p.572-583, 1993

BERGMANN, J.A.G.; ZAMBORLINI, L.C.; PROCÓPIO, C.S.O. et al. Estimativas de parâmetros genéticos do perímetro escrotal e do peso corporal em animais da raça Nelore. Arquivo Brasileiro de Medicina Veterinária e Zootecnia, v.48, n.1, p.69-78, 1996. 
BLASCO, A.; PILES, M.; VARONA, L. A Bayesian analysis of the effect of selection for growth rate on growth curves in rabbits. Genetics Selection Evolution, v.35, p.21-41, 2003.

BOURDON, R.M.; BRINKS, J.S. Scrotal circumference in yearling Hereford bulls: adjustment factors, heritabilities and genetic, environmental and phenotypic relationships with growth traits. Journal of Animal Science, v.62, p.958-967, 1986.

DAL FARRA, R.A.; FRIES, L.A.; LOBATO, J.F.P.Fatores de correção do perímetro escrotal para efeitos de idade e peso ao sobreano de tourinhos Nelore. Revista Brasileira de Zootecnia, v.27, n.6, p.1092-1096, 1998.

DIAS, L.T.; EL FARO, L.; ALBUQUERQUE, L. G. Estimativas de herdabilidade para perímetro escrotal de animais da raça Nelore. Revista Brasileira de Zootecnia, v.32, n.6, p.1878-1882. 2003 (supl.2).

ELER, J.P.; SILVA, J.A.V.; EVANS, J.L. et al. Additive genetic relationships between heifer pregnancy and scrotal circumference in Nellore cattle. Journal of Animal Science, v.82, p.2519-2527, 2004.

GAMERMAN, D. Simulação estocástica via cadeias de Markov. Caxambu: Associação Brasileira de Estatística, 1996. $196 p$.

GAMERMAN, D. Gibbs sampling. In: GAMERMAN, D. (Ed.) Markov chain Monte Carlo. London: Chapman \& Hall, 1997. p.119-159.

GELFAND, A.E.; SMITH, A.F.M. Sampling-based approaches to calculating marginal densities. Journal of the American Statistical Association, v.85, p.398-409, 1990.

MARTINS FILHO, R.; LÔBO, R.B. Estimates of genetic correlations between sire scrotal circumference and offspring age at first calving in Nelore cattle. Revista Brasileira de Genética, v.14, n.1, p.209-212, 1991 .

MEYER, K.; HAMMOND, K.; MACKINNON, M.J. et al. Estimates of covariances between reproduction and growth in Australian beef cattle. Journal of Animal Science, v.69, p.3533-3543, 1991.

ORTIZ PEÑA, C.D.; QUEIROZ, S.A.; FRIES, L.A. Comparação entre critérios de seleção de precocidade sexual e a associação destes com características de crescimento em bovinos Nelore. Revista Brasileira de Zootecnia, v.30, n.1, p.93-100, 2001.
PEREIRA, E.; ELER, J.A.; FERRAZ, J.B.S. et al. Correlação genética entre perímetro escrotal e algumas características reprodutivas na raça Nelore. Revista Brasileira de Zootecnia, v.29, n.6, p.1676-1683, 2000.

PINEDA, N.R.; FONSECA, V.O.; ALBUQUERQUE, L.G. Preliminar study of influence of scrotal circumference upon libido and seminal characteristics in young Nellore bulls. Arquivo Brasileiro de Medicina Veterinária e Zootecnia, v.52, n.1, p.69-75, 2000.

PRATT, S.L; SPITZER, J.C.; WEBSTER, H.W. et al. Comparison of methods for predicting yearling scrotal circumference and correlation of scrotal circumference to growth traits in beef bulls. Journal of Animal Science, v.69, p.2711-2720, 1991.

QUEIROZ, S.A.; PELICIONI,L.C.; SESANA, J.C. Estimativas de herdabilidade e correlação genética entre perímetro escrotal corrigido para idade e peso corporal de bovinos Caracu. In REUNIÃO ANUAL DA SOCIEDADE BRASILEIRA DE ZOOTECNIA, 38., 2001, Piracicaba. Anais... Piracicaba: Sociedade Brasileira de Zootecnia, 2001. p.524-525.

TEIXEIRA, R.A.; ALBUQUERQUE, L.G.; FRIES, L.A. Influência do peso à desmama, sobre medidas e avaliações do perímetro escrotal ao sobreano em touros Nelore. In: SIMPÓSIO NACIONAL DE MELHORAMENTO ANIMAL, 2., 1998 , Uberaba. Anais... Uberaba: Sociedade Brasileira de Melhoramento Animal, 1998. p.351-352.

Van KAAM, J.B.C.H.M. GIBANAL: analyzing program for Markov Chain Monte Carlo sequences version 2.3, 1997. (CD-ROM)

Van TASSELL, C.P.; van VLECK, D.L. A manual for use of MTGSAM. A set of FORTRAN programs to apply Gibbs sampling to animal models for variance component estimation [DRAFT]. Lincoln: U.S. Departament of Agriculture, Agricultural Research Service, 1995. 86p. 